\title{
Consumption of ultra-processed food and obesity: cross sectional results from the Brazilian Longitudinal Study of Adult Health (ELSA-Brasil) cohort (2008-2010)
}

\author{
Fernanda Marcelina Silva ${ }^{1}$, Luana Giatti ${ }^{2}$, Roberta Carvalho de Figueiredo ${ }^{3}$, \\ Maria del Carmen Bisi Molina ${ }^{4}$, Letícia de Oliveira Cardoso ${ }^{5}$, Bruce Bartholow Duncan 6 \\ and Sandhi Maria Barreto ${ }^{1,2, *}$ \\ ${ }^{1}$ Postgraduate Program in Sciences Applied to Adult Health Care, School of Medicine, Universidade Federal de \\ Minas Gerais, Belo Horizonte, MG, Brazil: ${ }^{2}$ Postgraduate Program in Public Health, School of Medicine, \\ Universidade Federal de Minas Gerais, Av. Alfredo Balena 190, Sala 814, CEP 30130100, Belo Horizonte, MG, \\ Brazil: ${ }^{3}$ Postgraduate Program in Health Sciences, Universidade Federal de São João Del-Rei, Divinópolis, MG, \\ Brazil: ${ }^{4}$ Postgraduate Program in Health and Nutrition, Universidade Federal do Espírito Santo, Vitória, ES, Brazil: \\ ${ }^{5}$ National School of Public Health, Fundação Oswaldo Cruz, Rio de Janeiro, RJ, Brazil: ${ }^{6}$ Postgraduate Program in \\ Epidemiology, School of Medicine, Universidade Federal Rio Grande do Sul, Porto Alegre, RS, Brazil
}

Submitted 11 September 2017: Final revision received 12 January 2018: Accepted 5 March 2018: First published online 12 April 2018

\begin{abstract}
Objective: To verify if the intake of ultra-processed foods is associated with higher BMI and waist circumference (WC) among participants of the Brazilian Longitudinal Study of Adult Health (ELSA-Brasil) cohort.

Design: Cross-sectional analysis of the ELSA-Brasil baseline (2008-2010). Dietary information obtained through an FFQ was classified according to characteristics of food processing (NOVA) and used to estimate the percentage energy contribution from ultra-processed foods (i.e. industrial formulations, elaborated from food processing, synthetic constituents and food additives) to individuals' total energy intake. BMI and WC and their respective cut-off points served as response variables. Associations were estimated through linear and multinomial logistic regression models, after adjusting for confounders and total energy intake.

Setting: Six Brazilian capital cities, 2008-2010.

Subjects: Active and retired civil servants, aged 35-64 years, from universities and research organizations ( $n$ 8977).

Results: Ultra-processed foods accounted for $22.7 \%$ of total energy intake. After adjustments, individuals in the fourth quartile of percentage energy contribution from ultra-processed foods presented $(\beta ; 95 \% \mathrm{CI})$ a higher BMI $(0.80 ; \mathrm{CI} 0.53$, $\left.1.07 \mathrm{~kg} / \mathrm{m}^{2}\right)$ and WC $(1.71 ; 1.02,2.40 \mathrm{~cm})$, and higher chances (OR; $\left.95 \% \mathrm{CI}\right)$ of being overweight $(1.31 ; 1.13,1.51)$, obese $(1.41 ; 1.18,1.69)$ and having significantly increased WC $(1.41 ; 1.20,1.66)$, compared with those in the first quartile. All associations suggest a dose-response gradient.

Conclusions: Results indicate the existence of associations between greater energy contribution from ultra-processed foods and higher BMI and WC, which are independent of total energy intake. These findings corroborate public policies designed to reduce the intake of this type of food.
\end{abstract}

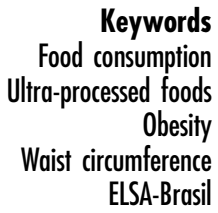

Obesity is an epidemic caused by a complex and multicausal process, which currently affects high-, middle- and low-income countries ${ }^{(1)}$. According to the WHO, in 2014, there were more than 1.9 billion overweight adults, of whom 600 million were obese ${ }^{(1)}$. The Global Burden of Disease Project estimated that, in 2015, high BMI was the world's fourth leading risk factor, largely contributing to the global burden of chronic non-communicable diseases and disabilities $^{(2)}$. In Brazil, the increase in obesity prevalence has been documented since the 1970s through national surveys and there has been a clear accelerated rise in the last two decades ${ }^{(3,4)}$.

In addition to greater incidence and prevalence of obesity, there has also been a marked increase in the production and consumption of ultra-processed foods ${ }^{(5,6)}$. Industrial processing creates 'food products' made from 
substances extracted from foods or obtained from foods or synthetic constituents and food additives. Ultra-processed foods have high energy density, high levels of $\mathrm{Na}$, free sugars and total, saturated and trans fats, as well as low levels of fibre, protein, micronutrients and phytochemicals. In addition, they are highly palatable, accessible and have a long shelf-life ${ }^{(7-9)}$. Some examples of ultraprocessed foods include hamburgers, sausages, cold meats, pizza, industrialized snacks, instant noodles, distilled beverages, diet and regular soda, candies, cereal bars and other fast-food meals.

Recent evidence in Brazil and other countries has shown an association between the intake or purchase of ultra-processed foods in general $^{(3,8,10)}$, or of specific ultra-processed foods ${ }^{(11-13)}$, and weight gain, overweight and obesity. Canella et al. $^{(3)}$ recently found a positive association between availability of ultra-processed foods at home and an increase in mean BMI and prevalence of overweight and obesity, using data from the Brazilian Household Budget Survey 2008-2009. By applying the module relative to individual dietary habits from this same survey (considering two $24 \mathrm{~h}$ food-intake registers), Louzada et $a l{ }^{(8)}$ also observed that a high ultra-processed food intake in adults and adolescents was associated with increased mean BMI and higher risk of overweight and obesity. A prospective study with adults participating in the SUN study (University of Navarra Follow-up) also demonstrated an association between ultra-processed food intake and a higher risk of overweight and obesity $^{(14)}$. However, these studies used only BMI as an anthropometric indicator of nutritional status.

Although BMI is a widely used measure in epidemiological studies for general adiposity, it has been considered a poor/insufficient indicator of obesity, since it is not able to separate muscle and adipose tissues or identify body fat distribution $^{(15-17)}$. Moreover, BMI is less associated with the risk of developing CVD and diabetes as compared with waist circumference (WC), which is an indicator frequently used to measure central adiposity ${ }^{(15)}$.

Thus, the objective of the present study was to investigate the association between intake of ultra-processed foods and BMI and WC among participants in the baseline of the Brazilian Longitudinal Study of Adult Health (ELSA-Brasil; 2008-2010). Our hypothesis is that a higher intake of ultraprocessed foods is associated with a higher BMI and WC.

\section{Methods}

The present study is a cross-sectional analysis of ELSABrasil baseline data, carried out between August 2008 and December 2010. ELSA-Brasil is a multicentre cohort comprising 15105 active or retired civil servants, aged 35-74 years, from universities (teaching and research organizations) in six Brazilian capital cities (states of Minas Gerais, São Paulo, Rio de Janeiro, Rio Grande do Sul, Espírito Santo and Bahia). Exclusion criteria for the ELSA-Brasil cohort were severe cognitive or communication impairment, intention to change job or stop working in the institution in the near future and, among those retired, residing outside the corresponding metropolitan area. Women with current or recent pregnancy were not included as such; their interview and examination were postponed to four or more months after delivery ${ }^{(18)}$. Further details about the study design, enrolment process and other information can be found in a previous study ${ }^{(18)}$. Details about the cohort profile were previously presented by Schmidt et al. ${ }^{(19)}$.

Out of 15105 ELSA-Brasil participants, forty-five were ineligible for the present study for not presenting information on dietary habits $(n$ 36) and/or anthropometric measurements, BMI ( $n$ 6) or WC ( $n$ 3). Exclusion criteria also included: (i) participants with an energy intake $\leq 1$ st percentile $(4796 \mathrm{~kJ} / \mathrm{d}(1146.3 \mathrm{kcal} / \mathrm{d}) ; n 150)$ or $\geq 99$ th percentile $(30028 \mathrm{~kJ} / \mathrm{d}(7176.9 \mathrm{kcal} / \mathrm{d}) ; n 151)$, due to the risk of under- or overestimating energy intake; (ii) participants who underwent bariatric surgery (regardless of the date of the surgery; $n$ 107) because of food intake alterations; (iii) individuals aged 65 years or over ( $n$ 1592) due to body composition changes observed in this age group regarding BMI and especially WC, which does not track central adiposity as well in older $v$. younger individuals $^{(20,21)}$; and (iv) participants who changed their dietary habits or went on a diet six months before the interview ( $n$ 4158). Therefore, the final sample was made up of 8977 participants. For other variables with missing data, exclusions took place only during the multivariate analysis.

For the present study, two response variables were used: BMI and WC, and both were analysed as continuous and categorical variables. BMI was obtained from weight and height $\left(\mathrm{kg} / \mathrm{m}^{2}\right)$ and the individuals were classified as eutrophic (BMI $<25 \cdot 0 \mathrm{~kg} / \mathrm{m}^{2}$ ), overweight (BMI $=25 \cdot 0$ $29.9 \mathrm{~kg} / \mathrm{m}^{2}$ ) and obese $\left(\mathrm{BMI} \geq 30 \cdot 0 \mathrm{~kg} / \mathrm{m}^{2}\right)^{(15)}$. Weight was measured on an electronic scale (Toledo ${ }^{\circledR}$, São Bernardo do Campo, São Paulo, Brazil) with 50 g precision. Height was measured using a stadiometer (Seca ${ }^{\circledR}$, Hamburg, Germany) with $0.1 \mathrm{~cm}$ precision.

WC was classified according to the WHO cut-off points as normal ( $<94 \mathrm{~cm}$ for men; $<80 \mathrm{~cm}$ for women), increased risk for metabolic complications $(\geq 94$ and $<102 \mathrm{~cm}$ for men; $\geq 80$ and $<88 \mathrm{~cm}$ for women) and significantly increased risk for metabolic complications $(\geq 102 \mathrm{~cm}$ for men; $\geq 88 \mathrm{~cm}$ for women) ${ }^{(15)}$, and herein called increased WC' and 'significantly increased WC', respectively. WC was measured at the mean point between the last rib and the iliac crest. For this measurement, a non-elastic anthropometric tape was used (Mabis ${ }^{\circledR}$, Waukegan, IL, USA) with $0 \cdot 1 \mathrm{~cm}$ precision.

All measurements were taken by a trained and certified research team, and the participants were fasted, with an empty bladder, wearing uniforms and barefoot ${ }^{(22)}$.

The explanatory variable was the percentage energy contribution from ultra-processed foods in total energy intake, which was grouped into quartiles. Information on 
dietary habits was obtained through a semi-quantitative FFQ with 114 food items, which measured dietary habits of the 12 months before the interview ${ }^{(23)}$. The FFQ presented satisfactory reliability for all nutrients and reasonable relative validity for energy, macronutrients, $\mathrm{Ca}$, $\mathrm{K}$, and vitamins $\mathrm{E}$ and $\mathrm{C}$. In the reproducibility analysis, the intraclass correlation coefficients varied between 0.55 and 0.83 for protein and vitamin $\mathrm{E}$, respectively. In the validation analysis, they varied between 0.20 and 0.72 for Se and Ca, respectively ${ }^{(24)}$.

The quantification of nutrients listed on the FFQ was carried out based on the following calculation: quantity of portions consumed per meal $\times$ weight/portion size $\times$ frequency of consumption $\times$ nutritional composition of the food portion. Nutritional composition of the food items was obtained through the Nutrition Data System for Research (NDSR) software from the University of Minnesota and based on the Brazilian Food Composition Database (TACO) from the Universidade Estadual de Campinas ${ }^{(23)}$.

Ultra-processed food intake was estimated using NOVA, a classification proposed by Monteiro et al. ${ }^{(7)}$, who divided foods according to their nature, extension and purpose of processing into four groups, as follows: Group 1, unprocessed or minimally processed foods; Group 2, processed culinary ingredients; Group 3, processed foods; and Group 4, ultra-processed foods ${ }^{(25)}$. In the ELSA-Brasil study, processed culinary ingredients were grouped with unprocessed or minimally processed foods ${ }^{(25)}$.

Ultra-processed foods were defined as industrial formulations elaborated from substances extracted from foods or obtained from foods or their synthetic constituents and food additives such as flavours, preservatives and sweeteners ${ }^{(7,8)}$. Compared with other food groups, these formulations are energy-dense and are higher in $\mathrm{Na}$, free sugars, total, saturated and trans fats, as well as lower in fibre and micronutrients. Examples of ultra-processed foods are shown in the online supplementary material, Supplemental Table 1.

Four variable groups were considered for adjustment: sociodemographic characteristics, socio-economic indicator, health-related behaviours and co-morbidities. Sociodemographic characteristics included sex, age and selfreported race/skin colour (white, brown, black, Asian or indigenous). The socio-economic indicator analysed was per capita family income (categorized into quintiles).

Health-related behaviours included smoking (never smoked, former smoker, smoker), leisure-time physical activity (low, moderate, vigorous), energy intake from unprocessed or minimally processed foods and processed culinary ingredients (Group $1+$ Group 2) and total energy intake. Energy intake from unprocessed or minimally processed foods and processed culinary ingredients was categorized into quartiles. Some examples of this group include fruits, vegetables, meats, rice, beans, salt and vegetable oils. Physical activity was measured using the dimension of leisure-time physical activity through the
International Physical Activity Questionnaire (IPAQ) and classified as low ( $<600$ MET min/week), moderate (600-3000 MET min/week) and vigorous ( $\geq 3000 \mathrm{MET}$ $\mathrm{min} /$ week), where MET is metabolic equivalent of $\operatorname{task}^{(26,27)}$.

As for co-morbidities used for adjustment, medical diagnosis report of diabetes mellitus (no and yes) and hypertension (no and yes) was obtained through the question: 'Has your doctor ever told you have had or have one of the following diseases?'. The possible answers were 'no', 'yes, only during pregnancy' and 'yes'. For the present study, the answer 'yes, only during pregnancy' was grouped with the option 'no'.

\section{Analysis}

First, a descriptive analysis of the study population was conducted with distribution of means and SD for continuous variables, and of proportions for categorical variables.

The magnitude of the association between ultraprocessed food intake and BMI and WC was estimated by multiple linear regression. Additionally, the association between ultra-processed food intake and categorized BMI and WC was estimated by multinomial logistic regression; in that, the reference categories were eutrophic and normal WC. Separate models were created for each response variable. Raw coefficients were estimated first, followed by consecutive adjustments for sociodemographic and socioeconomic characteristics, and health-related behaviours and co-morbidities. For clarity, the adjustments for Group 1 + Group 2 energy intake and total energy intake are shown separately in the two last models. Only the variables associated with the response variables at a significance level of $20 \%$ or less were included in the multivariate analysis. In the multivariate analyses, we used a significance level of $5 \%$ to retain a variable in the final model, except for sex, Group 1 + Group 2 energy intake and total energy intake that were kept regardless of their $P$ value. The adequacy of the linear regression models to the assumptions of homoscedasticity and normality was verified through graphs. The analyses were carried out using the statistical software package Stata version 12.1.

\section{Results}

Of 8977 participants, most were female (51.9\%), aged 45-54 years $(43.6 \%)$ and self-reportedly white (52.0\%; Table 1). According to BMI classification, 38.7\% of participants were overweight and $19.7 \%$ were obese. Regarding WC, $26.9 \%$ of participants were in the 'increased' and $31.0 \%$ in the 'significantly increased' risk categories for metabolic complications. Ultra-processed foods accounted for $22.7 \%$ of total energy intake.

Figure 1 shows the distribution of mean BMI (Fig. 1(a)) and mean WC (Fig. 1(b)), and their respective 95\% CI, adjusted for sex and age, according to ultra-processed food intake quartiles. The results suggest mean BMI and WC are greater as ultra-processed food intake increases. 
Table 1 Descriptive characteristics of the analytical sample according to quartile of the relative contribution of ultra-processed foods to total energy intake in the Brazilian Longitudinal Study of Adult Health (ELSA-Brasil), 2008-2010 ( $n$ 8977)

\begin{tabular}{|c|c|c|c|c|c|c|}
\hline & \multirow{2}{*}{$\begin{array}{l}\text { Overall population } \\
\% \text { or Median }\end{array}$} & \multicolumn{4}{|c|}{$\begin{array}{l}\text { Quartile of consumption of ultra-processed } \\
\text { foods (\% of total energy) }\end{array}$} & \multirow[b]{2}{*}{$P$ value } \\
\hline & & $\begin{array}{c}1 \mathrm{st} \\
(<16 \%)\end{array}$ & $\begin{array}{c}\text { 2nd } \\
(16-22 \%)\end{array}$ & $\begin{array}{c}3 r d \\
(22-29 \%)\end{array}$ & $\begin{array}{c}\text { 4th } \\
(>29 \%)\end{array}$ & \\
\hline $\operatorname{Sex}(\%)$ & & & & & & $<0.001$ \\
\hline Female & 51.9 & 21.6 & 23.6 & $26 \cdot 2$ & 28.6 & \\
\hline Male & $48 \cdot 1$ & $28 \cdot 7$ & 26.5 & 23.8 & $21 \cdot 0$ & \\
\hline Age group (\%) & & & & & & $<0.001$ \\
\hline $35-44$ years & $23 \cdot 6$ & $16 \cdot 4$ & $22 \cdot 9$ & $26 \cdot 1$ & $34 \cdot 6$ & \\
\hline $45-54$ years & $43 \cdot 6$ & 24.6 & $25 \cdot 2$ & $25 \cdot 8$ & $24 \cdot 4$ & \\
\hline $55-64$ years & $32 \cdot 8$ & $31 \cdot 7$ & $26 \cdot 3$ & $23 \cdot 1$ & $18 \cdot 9$ & \\
\hline Race/skin colour† (\%) & & & & & & $<0.001$ \\
\hline White & $52 \cdot 0$ & $18 \cdot 7$ & $23 \cdot 2$ & $27 \cdot 2$ & $30 \cdot 9$ & \\
\hline Brown & 28.5 & 30.5 & $27 \cdot 2$ & $22 \cdot 7$ & $19 \cdot 6$ & \\
\hline Black & $15 \cdot 2$ & $35 \cdot 0$ & $25 \cdot 9$ & $22 \cdot 1$ & $17 \cdot 1$ & \\
\hline Asian & $2 \cdot 3$ & 24.4 & 33.7 & 23.9 & $18 \cdot 1$ & \\
\hline Indigenous & 1.0 & $36 \cdot 8$ & 19.5 & $25 \cdot 3$ & $18 \cdot 4$ & \\
\hline Per capita family income† (\%) & & & & & & $<0.001$ \\
\hline 1st quintile & $14 \cdot 2$ & $18 \cdot 2$ & 23.5 & $25 \cdot 9$ & $32 \cdot 4$ & \\
\hline 2nd quintile & $23 \cdot 8$ & $19 \cdot 9$ & $25 \cdot 5$ & $25 \cdot 1$ & 29.5 & \\
\hline 3rd quintile & $19 \cdot 3$ & $23 \cdot 7$ & 24.9 & $26 \cdot 4$ & 24.9 & \\
\hline 4th quintile & $21 \cdot 3$ & $28 \cdot 2$ & 23.9 & $25 \cdot 1$ & $22 \cdot 8$ & \\
\hline 5th quintile & $21 \cdot 0$ & $33 \cdot 2$ & $26 \cdot 4$ & $23 \cdot 1$ & $17 \cdot 3$ & \\
\hline Group 1 + Group 2 intake $\neq$ (kJ; median) & 7579.7 & $8965 \cdot 5$ & $8132 \cdot 0$ & 7273.5 & 6073.9 & $<0.001$ \\
\hline Group 1+Group 2 intake (kcal; median) & $1811 \cdot 6$ & $2142 \cdot 8$ & $1943 \cdot 6$ & 1738.4 & $1451 \cdot 7$ & $<0.001$ \\
\hline Total energy intake (kJ; median) & 11634.4 & $11724 \cdot 4$ & 11824.4 & $11661 \cdot 6$ & $11368 \cdot 4$ & $<0.05$ \\
\hline Total energy intake (kcal; median) & $2780 \cdot 7$ & $2802 \cdot 2$ & $2826 \cdot 1$ & $2787 \cdot 2$ & $2717 \cdot 1$ & $<0.05$ \\
\hline Physical activity† (\%) & & & & & & 0.09 \\
\hline Low & 78.0 & 24.7 & 24.6 & 25.4 & $25 \cdot 3$ & \\
\hline Moderate & $14 \cdot 0$ & $27 \cdot 1$ & $26 \cdot 8$ & 23.6 & 22.5 & \\
\hline Vigorous & $6 \cdot 7$ & $25 \cdot 3$ & $25 \cdot 8$ & $22 \cdot 7$ & $26 \cdot 3$ & \\
\hline Smoking (\%) & & & & & & $<0.001$ \\
\hline Never smoked & $56 \cdot 6$ & $23 \cdot 0$ & 24.7 & $25 \cdot 8$ & $26 \cdot 6$ & \\
\hline Former smoker & $28 \cdot 1$ & $27 \cdot 3$ & $25 \cdot 6$ & 24.0 & 23.0 & \\
\hline Smoker & 15.4 & $28 \cdot 1$ & $25 \cdot 2$ & 23.8 & $22 \cdot 9$ & \\
\hline Hypertension† (\%) & & & & & & $<0.001$ \\
\hline No & 71.3 & $23 \cdot 1$ & $25 \cdot 2$ & $25 \cdot 4$ & $26 \cdot 3$ & \\
\hline Yes & 28.7 & $29 \cdot 8$ & 24.5 & $24 \cdot 0$ & 21.7 & \\
\hline Diabetes mellitus† (\%) & & & & & & $<0.001$ \\
\hline No & 93.8 & $23 \cdot 8$ & $25 \cdot 1$ & 25.4 & $25 \cdot 7$ & \\
\hline Yes & $6 \cdot 2$ & $43 \cdot 1$ & 23.6 & $19 \cdot 0$ & $14 \cdot 3$ & \\
\hline
\end{tabular}

†There may be differences in totals due to loss of information.

¥Combined intake of unprocessed or minimally processed foods (Group 1) and processed culinary ingredients (Group 2).

The associations between ultra-processed food consumption and BMI and WC are depicted in Table 2. In the univariate analysis, a tendency of greater BMI as ultra-processed food intake increased was observed, but this association was not statistically significant $(P=0 \cdot 21)$. When adjustments were made for sociodemographic and socio-economic characteristics, these associations became statistically significant: individuals in the fourth ultra-processed food intake quartile presented a mean BMI $0.83(95 \%$ CI $0.55,1.11) \mathrm{kg} / \mathrm{m}^{2}$ higher than those in the first quartile, with a dose-response gradient between the associations. These associations remained after adjustment for health-related behaviours and co-morbidities, as did the dose-response gradient. We observed that individuals in the fourth quartile of ultra-processed food intake presented a mean BMI $0 \cdot 80$ (95\% CI $0.53,1.07) \mathrm{kg} / \mathrm{m}^{2}$ higher than the individuals in the first quartile.
Regarding WC, the univariate analysis showed a negative and decreasing association as ultra-processed food intake increased. The individuals in the fourth quartile presented a mean WC $1.11(95 \% \mathrm{CI}-1.84,-0.37) \mathrm{cm}$ lower than those in the first quartile. However, when adjustments were made for sociodemographic and socio-economic characteristics, these associations became positive and statistically significant, with a gradual increase between the quartiles. As compared with the individuals in the first quartile, those in the fourth ultra-processed food intake quartile presented a mean WC 1.82 (95\% CI 1.11, 2.54) $\mathrm{cm}$ higher. After adjustments for sociodemographic and socioeconomic characteristics, health-related behaviours and comorbidities, we observed that individuals in the fourth ultra-processed food intake quartile presented mean WC 1.71 (95\% CI 1.02, 2.40) cm higher when compared with individuals in the first quartile, with the presence of a dose-response gradient between the associations (Table 2). 

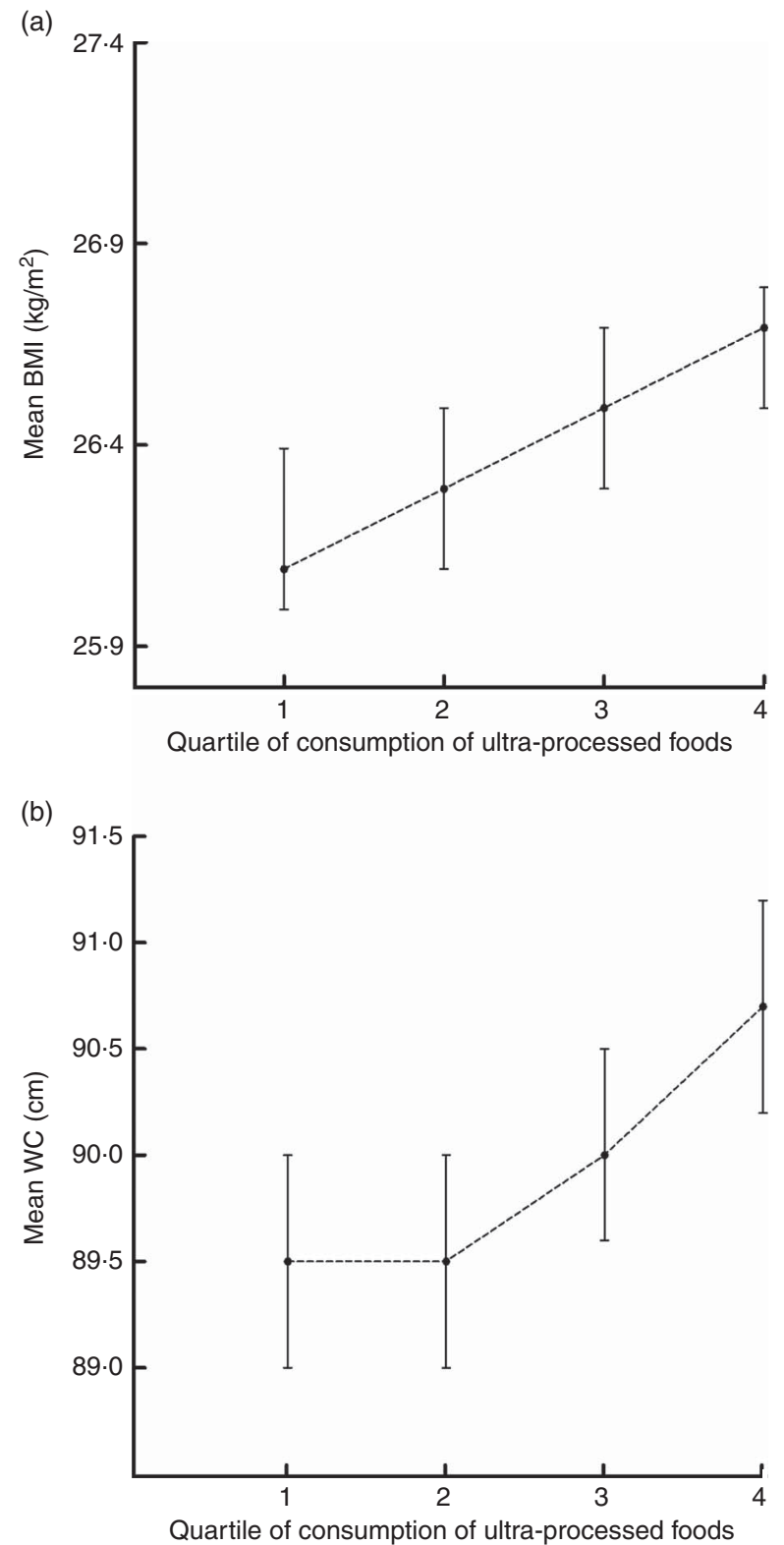

Fig. 1 Distribution of (a) mean BMI and (b) mean waist circumference (WC) according to quartile of the relative contribution of ultra-processed foods to total energy intake in the Brazilian Longitudinal Study of Adult Health (ELSA-Brasil), 2008-2010 ( $n$ 8977). Values are means, with their 95\% Cl represented by vertical bars, adjusted for sex and age (both $P$ trend $<0.01$ )

Table 3 shows the results of the multinomial logistic regression models for the association between ultraprocessed food intake and overweight, obesity and WC categories. After all adjustments, the individuals in the fourth quartile, as compared with those in the first quartile and having the eutrophic participants as reference, had $31 \%(\mathrm{OR}=1.31 ; 95 \% \mathrm{CI} 1.13,1.51)$ and $41 \%(\mathrm{OR}=1.41$; $95 \%$ CI $1.18,1.69)$ greater odds to be overweight and obese, respectively, with dose-response gradients in the associations. Regarding WC, after all adjustments, compared with individuals with normal WC, those in the fourth quartile of ultra-processed food intake had $41 \%$ greater odds of presenting a significantly increased WC $(\mathrm{OR}=1.41 ; 95 \% \mathrm{CI} 1.20,1.66)$ compared with those in the first quartile. No association was found between ultra-processed food intake and the increased WC category (Table 3 ).

Further adjustments for the consumption of unprocessed or minimally processed foods and processed culinary ingredients (Group $1+$ Group) and total energy intake (final models of Tables 2 and 3) attenuated the magnitude of the associations but they remained in the same direction and statistically significant.

\section{Discussion}

The present study stands out for investigating the association between BMI and WC and ultra-processed food intake. As far as we know, it is also the first to show an association between WC and ultra-processed food intake according to the classification proposed by Monteiro et $a l .^{(7)}$.

After adjustments for sociodemographic characteristics, socio-economic indicator, health-related behaviours and co-morbidities, greater BMI and WC were observed as ultra-processed food intake increased. Similar results were obtained in the analysis using the cut-off points for overweight and obesity, as well as for significantly increased WC. These results remained positive even after adjustment for consumption of unprocessed or minimally processed foods and processed culinary ingredients (Group $1+$ Group 2) and after adjustment for total energy intake.

Our results reinforce the hypothesis that not only the amount of ingested energy contributes to weight gain, but also the nature of this energy, as the associations found are independent of the level of energy intake. It is also worth pointing out that our explanatory variable was the percentage contribution from ultra-processed foods to total energy intake; that is, individuals with low total energy intake $(<6276 \mathrm{~kJ}(<1500 \mathrm{kcal})$ daily $)$ and those with high energy intake ( $>18828 \mathrm{~kJ}$ ( $>4500 \mathrm{kcal})$ daily) would be both grouped in the fourth quartile of the explanatory variable if ultra-processed foods accounted for $29 \%$ or more of their total energy intake. Consequently, the adjustment for total energy intake may be regarded as an over-adjustment and, if such, the magnitudes of the associations are higher, as shown in the models of Tables 2 and 3.

The associations found in the present study can be partly explained by the characteristics of these foods, such as accessibility, practicality and high palatability, which encourage the exaggerated and 'unnoticed' consumption of these foods ${ }^{(28)}$. Moreover, these foods present poor nutritional value due to their high energy density, high levels of $\mathrm{Na}$, free sugars, total, saturated and trans fats; their low levels of fibre and micronutrients; and the presence of artificial flavourings, preservatives and other additives $^{(7,29)}$. 
Table 2 Unadjusted and adjusted coefficients $(\beta)$ of the associations between the relative contribution of ultra-processed foods to total energy intake (in quartiles) and BMI and waist circumference in the Brazilian Longitudinal Study of Adult Health (ELSA-Brasil), 2008-2010 ( $n$ 8977)

\begin{tabular}{|c|c|c|c|c|c|c|c|}
\hline & \multicolumn{7}{|c|}{ Quartile of consumption of ultra-processed foods (\% of total energy) } \\
\hline & \multirow[t]{2}{*}{$1 \mathrm{st}$} & \multicolumn{2}{|c|}{ 2nd } & \multicolumn{2}{|c|}{ 3rd } & \multicolumn{2}{|c|}{ 4th } \\
\hline & & $\beta$ & $95 \% \mathrm{Cl}$ & $\beta$ & $95 \% \mathrm{Cl}$ & $\beta$ & $95 \% \mathrm{Cl}$ \\
\hline \multicolumn{8}{|l|}{ BMI } \\
\hline Model $0 \dagger$ & Reference & 0.01 & $-0.26,0.27$ & 0.10 & $-0.17,0.37$ & 0.17 & $-0.10,0.44$ \\
\hline Model $1 \ddagger$ & Reference & $0.30^{\star}$ & $0.03,0.57$ & $0.53^{\star \star \star}$ & $0.25,0.80$ & $0.83^{\star * \star}$ & $0.55,1.11$ \\
\hline Model $2 \S$ & Reference & $0.41^{\star *}$ & $0.15,0.67$ & $0.55^{\star \star \star}$ & $0.28,0.81$ & $0.80^{\star \star \star}$ & $0.53,1.07$ \\
\hline Model $3 \|$ & Reference & $0.43^{\star *}$ & $0.17,0.69$ & $0.59^{\star \star \star}$ & $0.33,0.86$ & $0.89^{\star \star \star}$ & $0.61,1.17$ \\
\hline Model 4ள & Reference & $0.34^{*}$ & $0.08,0.61$ & $0.44^{\star \star}$ & $0.17,0.72$ & $0.64^{\star \star \star}$ & $0.33,0.95$ \\
\hline \multicolumn{8}{|c|}{ Waist circumference } \\
\hline Model $0 \dagger$ & Reference & -0.71 & $-1.45,0.02$ & $-0.89^{*}$ & $-1 \cdot 62,-0.15$ & $-1 \cdot 11^{\star \star}$ & $-1.84,-0.37$ \\
\hline Model 1‡ & Reference & 0.40 & $-0.29,1.09$ & $1.00^{\star *}$ & $0.30,1 \cdot 70$ & $1.82^{\star \star \star}$ & $1 \cdot 11,2 \cdot 54$ \\
\hline Model $2 \S$ & Reference & 0.65 & $-0.02,1.31$ & $1.02^{\star \star}$ & $0.35,1.70$ & $1.71^{* \star *}$ & $1.02,2.40$ \\
\hline Model 3\| & Reference & $0.73^{\star}$ & $-0.02,1.31$ & $1 \cdot 22^{\star \star \star}$ & $0.35,1.70$ & $2 \cdot 10^{\star \star \star}$ & $1.02,2.40$ \\
\hline Model 4" & Reference & 0.34 & $-0.34,1.01$ & 0.53 & $-0.18,1.24$ & $0.95^{\star}$ & $0.17,1.74$ \\
\hline
\end{tabular}

${ }^{\star} P<0.05,{ }^{* *} P<0.01,{ }^{* * *} P<0.001$.

†Model 0: unadjusted.

¥Model 1: Model $0+$ sex, age, race/skin colour and per capita family income.

§Model 2: Model $0+$ Model $1+$ physical activity, smoking, hypertension and diabetes.

$\|$ Model 3: Model 0+ Model 1+ physical activity, smoking, hypertension, diabetes and Group $1+$ Group 2 energy intake.

IModel 4: Model 0+ Model 1 + physical activity, smoking, hypertension, diabetes, Group $1+$ Group 2 energy intake and total energy intake.

Table 3 Unadjusted and adjusted odds of the associations between the relative contribution of ultra-processed foods to total energy intake (in quartiles) and overweight, obesity, increased and significantly increased waist circumference in the Brazilian Longitudinal Study of Adult Health (ELSA-Brasil), 2008-2010 ( $n$ 8977)

\begin{tabular}{|c|c|c|c|c|c|c|c|}
\hline & \multicolumn{7}{|c|}{ Quartile of consumption of ultra-processed foods ( $\%$ of total energy) } \\
\hline & \multirow[t]{2}{*}{$1 \mathrm{st}$} & \multicolumn{2}{|c|}{ 2nd } & \multicolumn{2}{|c|}{ 3rd } & \multicolumn{2}{|c|}{ 4th } \\
\hline & & OR & $95 \% \mathrm{Cl}$ & OR & $95 \% \mathrm{Cl}$ & OR & $95 \% \mathrm{Cl}$ \\
\hline \multicolumn{8}{|l|}{ Overweight } \\
\hline Model $0 \dagger$ & Reference & 0.97 & $0.85,1.11$ & 1.01 & $0.89,1.16$ & 0.97 & $0.85,1.11$ \\
\hline Model $1 \ddagger$ & Reference & 1.09 & $0.96,1.25$ & $1.23^{\star *}$ & $1.08,1.41$ & $1 \cdot 32^{\star \star *}$ & $1.15,1.52$ \\
\hline Model $2 \S$ & Reference & $1 \cdot 12$ & $0.98,1.29$ & $1 \cdot 22^{\star \star}$ & $1.06,1.40$ & $1.31^{\star \star \star}$ & $1.13,1.51$ \\
\hline Model $3 \|$ & Reference & 1.14 & $0.99,1.30$ & $1.24^{* *}$ & $1.07,1.42$ & $1 \cdot 32^{\star \star \star}$ & $1.15,1.53$ \\
\hline Model 4" & Reference & 1.14 & $0.99,1.31$ & $1.24^{\star *}$ & $1.08,1.42$ & $1.32^{\star * *}$ & $1.15,1.53$ \\
\hline \multicolumn{8}{|l|}{ Obesity } \\
\hline Model $0 \dagger$ & Reference & 0.94 & $0.80,1.11$ & 1.02 & $0.87,1.20$ & 1.02 & $0.87,1.19$ \\
\hline Model $1 \ddagger$ & Reference & $1 \cdot 10$ & $0.93,1.30$ & $1.29^{\star \star}$ & $1.09,1.53$ & $1.45^{\star \star \star}$ & $1.23,1.73$ \\
\hline Model $2 \S$ & Reference & $1 \cdot 15$ & $0.97,1.37$ & $1 \cdot 27^{\star \star}$ & $1.07,1.51$ & $1.41^{\star \star *}$ & $1.18,1.69$ \\
\hline Model 3 & Reference & 1.19 & $1.00,1.42$ & $1.32^{* \star}$ & $1.10,1.57$ & $1.46^{\star \star *}$ & $1.22,1.75$ \\
\hline Model 4" & Reference & $1 \cdot 17$ & $0.98,1.39$ & $1 \cdot 30^{\star \star}$ & $1.09,1.55$ & $1.43^{\star \star \star}$ & $1.20,1.72$ \\
\hline \multicolumn{8}{|c|}{ Increased waist circumference } \\
\hline Model $0 \dagger$ & Reference & $1 \cdot 10$ & $0.95,1.27$ & 1.08 & $0.93,1.25$ & 1.03 & $0.89,1.19$ \\
\hline Model $1 \ddagger$ & Reference & $1 \cdot 16^{\star}$ & $1.00,1.34$ & $1 \cdot 15$ & $0.99,1.34$ & $1 \cdot 13$ & $0.97,1.32$ \\
\hline Model $2 \S$ & Reference & $1 \cdot 19^{*}$ & $1.02,1.38$ & $1 \cdot 16$ & $0.99,1.35$ & 1.13 & $0.96,1.32$ \\
\hline Model 3\| & Reference & $1 \cdot 19^{*}$ & $1.02,1.38$ & 1.16 & $1.00,1.36$ & 1.13 & $0.96,1.34$ \\
\hline Model 4" & Reference & 1.14 & $0.98,1.33$ & 1.08 & $0.92,1.27$ & 1.00 & $0.84,1.20$ \\
\hline \multicolumn{8}{|c|}{ Significantly increased waist circumference } \\
\hline Model 0† & Reference & 1.02 & $0.89,1.17$ & 1.11 & $0.97,1.28$ & 1.13 & $0.98,1.29$ \\
\hline Model $1 \ddagger$ & Reference & $1 \cdot 15$ & $0.99,1.33$ & $1.29^{* *}$ & $1.11,1.50$ & $1.42^{\star \star *}$ & $1.22,1.65$ \\
\hline Model $2 \S$ & Reference & $1.23^{*}$ & $1.05,1.43$ & $1.32^{* *}$ & $1.13,1.54$ & $1.41^{\star \star *}$ & $1.20,1.66$ \\
\hline Model $3 \|$ & Reference & $1 \cdot 25^{\star *}$ & $1.07,1.46$ & $1 \cdot 38^{\star * \star}$ & $1.18,1.62$ & $1.55^{\star \star *}$ & $1.31,1.83$ \\
\hline Model 4đ & Reference & $1 \cdot 14$ & $0.98,1.35$ & $1 \cdot 19^{\star}$ & $1.01,1.40$ & $1 \cdot 21^{*}$ & $1.01,1.46$ \\
\hline
\end{tabular}

Reference categories are eutrophic (for overweight and obesity) and normal waist circumference (for increased and significantly increased waist circumference). ${ }^{*} P<0.05,{ }^{* \star} P<0.01,{ }^{* * \star} P<0.001$.

†Model 0: unadjusted.

†Model 1: Model $0+$ sex, age, race/skin colour and per capita family income.

§Model 2: Model $0+$ Model 1 + physical activity, smoking, hypertension and diabetes.

$\|$ Model 3: Model 0 + Model 1 + physical activity, smoking, hypertension, diabetes and Group $1+$ Group 2 energy intake.

IModel 4: Model 0 + Model 1 + physical activity, smoking, hypertension, diabetes, Group $1+$ Group 2 energy intake and total energy intake. 
The lack of association between ultra-processed food intake and BMI, overweight and obesity, observed in the univariate analysis, may be explained by the presence of multiple confounding factors that interfere in this association in opposite directions. In the present study, the variable age, for instance, seems to play this role, since after adjusting for age we found statistically significant associations between the variables. Regarding WC, a negative and decreasing association between increased ultra-processed food intake and WC was found in the univariate analysis. However, after adjusting for age, this association became positive and increasing, indicating a strong negative confounding factor. Previous studies conducted in Brazil showed higher intake of ultraprocessed foods among younger individuals ${ }^{(4,30)}$.

The results of the present study corroborate those described by Canella et al. ${ }^{(3)}$, who found a positive association between the availability of ultra-processed foods at home and the prevalence of overweight and obesity in Brazilians. However, Louzada et $a l^{(8)}$, who evaluated individual food consumption by means of two $24 \mathrm{~h}$ foodintake registers, found a positive association between ultra-processed food intake and BMI, overweight and obesity only in women. The authors suggest that the lack of association in males may result from confounding factors that were not measured, or measured with higher error in this group, such as the variables physical activity and smoking, which were indirectly estimated. It is unlikely that this happened in the present study, considering all adjustment variables used were collected through interviews or examinations, with a high level of rigour and quality control and assurance, with no quality differential for men and women ${ }^{(22)}$. It is noteworthy that the instrument used to evaluate the participants' food consumption was different from that used for ELSA-Brasil. This explains some of the differences found between the two studies, since FFQ are generally more appropriate to assess usual food consumption, because food diaries generally do not cover an appropriate number of days and require correction for intra-individual variance to be used. In addition, FFQ are easy, quick and low cost, and have the advantage of obtaining overall information on intakes over a long period of time $e^{(31,32)}$

The consistent findings of the current study are reinforced by the results of previous studies from different countries. A study carried out by the Pan American Health Organization, in Latin American countries between 2000 and 2013, found a positive association between the sales of ultra-processed foods and obesity in adults ${ }^{(10)}$. In a cohort with young adults in the USA, a strong positive association was found between the habit of eating at fastfood restaurants, which mostly sell ultra-processed foods, and weight gain $^{(13)}$. Similarly, another study involving three cohorts reported a positive association between ultra-processed foods, such as French fries, chips, processed meats, soft drinks and trans fat, and long-term weight gain ${ }^{(11)}$. Furthermore, prospective and crosssectional studies, performed in different countries, indicate a strong association between the elevated consumption of soft drinks and weight gain and obesity ${ }^{(12,33-35)}$. However, unlike some previous studies, a study conducted in the UK using data from the National Diet and Nutrition Survey (2008-2012) described no association between ultra-processed food intake and body weight ${ }^{(36)}$.

Although we did not find other studies that evaluated the relationship between ultra-processed food intake, as defined by NOVA, and WC, some investigations corroborate our results. Cunha et al. ${ }^{(37)}$ analysed data from adults living in the metropolitan region of Rio de Janeiro and demonstrated a positive association between WC and western dietary habits, characterized by fast foods, sweets and soft drinks, in women.

A longitudinal analysis with data from five European countries participating in the European Prospective Investigation into Cancer and Nutrition showed a positive association between the consumption of bread, processed meat, margarine and soft drinks, and WC elevation in the periods analysed, after adjustment for BMI and WC in the study baseline ${ }^{(38)}$.

The results of analyses using data from the Framingham Heart Study showed that individuals who consumed one $(350 \mathrm{ml})$ or more cans of soft drinks per day presented a higher risk of developing an increased WC $(\geq 102 \mathrm{~cm}$ for men and $\geq 88 \mathrm{~cm}$ for women) and other metabolic syndrome components, as compared with those having infrequent consumption (less than one can per day) $)^{(35)}$.

Some studies ${ }^{(34,39)}$ suggest that the presence of fructoserich corn syrup in these beverages favours weight gain and abdominal obesity. However, results found by Dhingra et al. ${ }^{(35)}$ showed no difference in associations considering regular or diet beverages, which suggests the influence of other factors in this relationship. It is important to remember that liquid foods have lower satiety power and can thus lead to an exaggerated energy intake $\mathrm{e}^{(12,34,35)}$.

We must consider that the classification of foods according to nature, extension and processing purpose is recent and still under development, and some changes may still be incorporated to NOVA with time. In contrast to traditional systems of food classification, this new system does not focus on nutrients but rather on the nature, extension and processing purposes, making distinctions between types, uses and effects of food processing ${ }^{(40,41)}$, representing a new view of what matters most as we face the era of obesity and chronic diseases. On the other hand, although it may appear simple to use, the NOVA system requires that people know and report correctly what they eat in order to apply this food classification, and this may lead to non-differential misclassification of some foods ${ }^{(40)}$. We believe that the NOVA classification opens up new avenues to understand the role of industrialized diets in the development of the most common chronic diseases 
and will help to improve public policies towards the control of the obesity epidemic ${ }^{(41)}$.

The present study stands out for investigating the association between ultra-processed food intake and WC in a sample of Brazilian adults; for assessing individual food intake through a validated $\mathrm{FFQ}^{(23,24)}$; use of the NOVA classification which, despite being new, helps us understand the role of dietary habits in the obesity epidemic; and also for the sample size and sample diversity. Moreover, it is worth highlighting that association estimates were adjusted for important confounding factors.

Nevertheless, some limitations must be acknowledged. Considering its cross-sectional design, it is not possible to establish causality between ultra-processed food intake and BMI and WC. Although the ELSA-Brasil cohort is not representative of the Brazilian population, there is no reason to doubt that the associations found can be extrapolated to similar adult populations, considering we did not identify factors that modify the associations shown ${ }^{(42)}$. We must consider that the classification of foods according to nature, extension and processing purpose is recent and can therefore be reviewed in the future. Furthermore, the FFQ used with the participants in the baseline was not designed to classify foods according to characteristics of food processing; therefore, non-differential classification errors may occur, which would lead to an underestimation of the magnitude of associations found ${ }^{(43)}$. In addition, the FFQ favours an overestimation of food consumption ${ }^{(44)}$; however, this is true of all groups and not only for ultraprocessed foods. Also, considering we used the percentage mean daily energy intake attributed to ultra-processed foods, as opposed to specific quantities, it is unlikely that any overestimation due to FFQ limitation interfered in our results. Finally, because BMI and WC are very highly correlated ( $r=85 \%$ ), we could not adjust the analysis of WC for BMI, and thus we cannot guarantee that the associations of ultra-processed foods with WC are independent of BMI.

\section{Conclusion}

The present study has shown that a higher ultra-processed food intake is associated with higher BMI and WC independently of the level of energy intake, after adjustments for confounding variables, with dose-response gradient between associations. These findings corroborate the hypothesis that links the increase in ultra-processed food intake with the obesity epidemic in Brazil and worldwide. Considering food selection and supply are highly impacted by the sociocultural, economic and health environment, our results reinforce the importance of public policies such as the Dietary Guidelines for the Brazilian Population, revised in $2014^{(45)}$, which promote the reduction of ultra-processed food consumption and guide the population to replace them for unprocessed and minimally processed foods.

\section{Acknowledgements}

Acknowledgements: The authors thank the staff and participants of the ELSA-Brasil for their important contributions. Financial support: The ELSA-Brasil baseline study is supported by the Brazilian Ministry of Health, Science and Technology Department (DECIT); and the Brazilian Ministry of Science and Technology and Innovation, Financiadora de Estudos e Projetos (FINEP) and Conselho Nacional de Desenvolvimento Científico e Tecnológico (CNPq) (grant numbers 01060010.00 RS, 01060212.00 BA, 01060300.00 ES, 01060278.00 MG, $01060115.00 \mathrm{SP}$ and 01060071.00 RJ). F.M.S received a master's scholarship from the Coordenação de Aperfeiçoamento de Pessoal de Nível Superior (CAPES). S.M.B., L.G., M.D.C.B.M. and B.B.D. are research fellows of CNPq. DECIT, FINEP, CNPq and CAPES had no role in the design, analysis or writing of this article. Conflict of interest: None. Authorship: F.M.S., S.M.B., L.G. and R.C.F. contributed to study conception, analysis and interpretation of data, manuscript drafting and critical manuscript revision for important intellectual content. M.D.C.B.M., L.O.C. and B.B.D. contributed to critical review of the manuscript for important intellectual content. Ethics of buman subject participation: This study was conducted according to the guidelines laid down in the Declaration of Helsinki and approved by the Committee of Ethics in Research (approval number 189/2006). Written informed consent was obtained from all participants.

\section{Supplementary material}

To view supplementary material for this article, please visit https://doi.org/10.1017/S1368980018000861

\section{References}

1. World Health Organization (2016) Obesity and overweight. Fact sheet no. 311. http://www.who.int/mediacentre/ factsheets/fs311/en/\# (accessed June 2016).

2. GBD 2015 Risk Factors Collaborators (2016) Global, regional, and national comparative risk assessment of 79 behavioural, environmental and occupational, and metabolic risks or clusters of risks, 1990-2015: a systematic analysis for the Global Burden of Disease Study 2015. Lancet 388, 1659-1724.

3. Canella DS, Levy RB, Martins AP et al. (2014) Ultra-processed food products and obesity in Brazilian households (2008-2009). PLoS One 9, e92752.

4. Brazilian Institute of Geography and Statistics (2011) Search for Family Budgets 2008-2009: Analysis of Personal Food Consumption in Brazil. Rio de Janeiro, RJ: IBGE.

5. Juul F \& Hemmingsson E (2015) Trends in consumption of ultra-processed foods and obesity in Sweden between 1960 and 2010. Public Health Nutr 18, 3096-3107.

6. Monteiro CA, Moubarac J-C, Cannon G et al. (2013) Ultra-processed products are becoming dominant in the global food system. Obes Rev 14, 21-28.

7. Monteiro CA, Cannon G, Levy RB et al. (2016) NOVA. The star shines bright. World Nutr 7, 28-38. 
8. Louzada ML, Baraldi LG, Steele EM et al. (2015) Consumption of ultra-processed foods and obesity in Brazilian adolescents and adults. Prev Med 81, 9-15.

9. Bielemann RM, Motta JV, Minten GC et al. (2015) Consumption of ultra-processed foods and their impact on the diet of young adults. Rev Saude Publica 49, 28.

10. Pan American Health Organization (2015) Ultra-Processed Food and Drink Products in Latin America: Trends, Impact on Obesity, Policy Implications. Washington, DC: PAHO.

11. Mozaffarian D, Hao T, Rimm EB et al. (2011) Changes in diet and lifestyle and long- term weight gain in women and men. $N$ Engl J Med 364, 2392-2404.

12. Hu FB \& Malik VS (2010) Sugar-sweetened beverages and risk of obesity and type 2 diabetes: epidemiologic evidence. Physiol Behav 100, 47-54.

13. Pereira MA, Kartashov AI, Ebbeling CB et al. (2005) Fastfood habits, weight gain, and insulin resistance (the CARDIA study): 15-year prospective analysis. Lancet 365, 36-42.

14. Mendonça RD, Pimenta AM, Gea A et al. (2016) Ultraprocessed food consumption and risk of overweight and obesity - the University of Navarra Follow-Up (SUN) cohort study. Am J Clin Nutr 104, 1433-1440.

15. World Health Organization (2000) Obesity: Preventing and Managing the Global Epidemic. Report of a WHO Consultation. WHO Technical Report Series no. 894. Geneva: WHO.

16. Wellens RI, Roche AF, Khamis HJ et al. (1996) Relationships between the body mass index and body composition. Obes Res 4, 35-44.

17. Flegal KM, Shepherd JA, Looker AC et al. (2009) Comparisons of percentage body fat, body mass index, waist circumference, and waist-stature ratio in adults. Am J Clin Nutr 89, 500-508.

18. Aquino EM, Barreto SM, Benseñor IM et al. (2012) Brazilian Longitudinal Study of Adult Health (ELSA-Brasil): objectives and design. Am J Epidemiol 175, 315-324.

19. Schmidt MI, Duncan BB, Mill JG et al. (2015) Cohort profile: Longitudinal Study of Adult Health (ELSA-Brasil). Int $J$ Epidemiol 44, 68-75.

20. Zamboni M, Rossi AP, Fantin F et al. (2013) Adipose tissue, diet and aging. Mech Ageing Dev 136-137, 129-137.

21. Heymsfield S, Lohman T, Wang ZM et al. (2005) Human Body Composition, 2nd ed. Champaign, IL: Human Kinetics Publishers.

22. Schmidt MI, Griep RH, Passos VM et al. (2013) Estratégias e desenvolvimento de garantia e controle de qualidade no ELSA-Brasil. Rev Saude Publica 47, Suppl. 2, 105-112.

23. Molina MDCB, Faria CP, Cardoso LO et al. (2013) Diet assessment in the Brazilian Longitudinal Study of Adult Health (ELSA-Brasil): development of a food frequency questionnaire. Rev Nutr 26, 167-176.

24. Molina MDCB, Benseñor IM, Cardoso LO et al. (2013) Reproducibility and relative validity of the food frequency questionnaire used in the ELSA-Brasil. Cad Saude Publica 29, 369-379.

25. Simões BS, Cardoso LO, Benseñor IM et al. (2018) Consumption of ultra-processed foods and socioeconomic position: a cross-sectional analysis of the Brazilian Longitudinal Study of Adult Health. Cad Saude Publica 34, e00019717.

26. Craig CL, Marshall AL, Sjöström M et al. (2003) International physical activity questionnaire: 12-country reliability and validity. Med Sci Sports Exerc 35, 1381-1395.
27. International Physical Activity Questionnaire (2005) Guidelines for Data Processing and Analysis - Short and Long Forms. http://www.ipaq.ki.se/ (accessed November 2016).

28. Ludwig DS (2011) Technology, diet, and the burden of chronic disease. JAMA 305, 1352-1353.

29. Monteiro CA (2009) Nutrition and health. The issue is not food, nor nutrients, so much as processing. Public Health Nutr 12, 729-731.

30. Cardoso LO, Carvalho MS, Cruz OG et al. (2016) Eating patterns in the Brazilian Longitudinal Study of Adult Health (ELSA-Brasil): an exploratory analysis. Cad Saude Publica 32, e00066215.

31. Kowalkowska J, Slowinska MA, Slowinski D et al. (2013) Comparison of a full food-frequency questionnaire with the three-day unweighted food records in young Polish adult women: implications for dietary assessment. Nutrients 5, 2747-2776.

32. Slater B, Philippi ST, Marchioni DML et al. (2003) Validation of food frequency questionnaires - FFQ: methodological considerations. Rev Bras Epidemiol 6, 200-208.

33. Malik VS, Pan A, Willett WC et al. (2013) Sugar-sweetened beverages and weight gain in children and adults: a systematic review and meta-analysis. Am J Clin Nutr 98, $1084-1102$.

34. Te Morenga L, Mallard S \& Mann J (2012) Dietary sugars and body weight: systematic review and meta-analyses of randomised controlled trials and cohort studies. BMJ 346, e7492.

35. Dhingra R, Sullivan L, Jacques PF et al. (2007) Soft drink consumption and risk of developing cardiometabolic risk factors and the metabolic syndrome in middle-aged adults in the community. Circulation 116, 480-488.

36. Adams J \& White M (2015) Characterisation of UK diets according to degree of food processing and associations with socio-demographics and obesity: cross-sectional analysis of UK National Diet and Nutrition Survey (2008-12). Int J Behav Nutr Phys Act 12, 160.

37. Cunha DB, Almeida RM, Sichieri R et al. (2010) Association of dietary patterns with BMI and waist circumference in a low-income neighbourhood in Brazil. Br J Nutr 104, 908-913.

38. Romaguera D, Ängquist L, Du H et al. (2011) Food composition of the diet in relation to changes in waist circumference adjusted for body mass index. PLoS One $\mathbf{6}$, e23384.

39. Mirmiran P, Yuzbashian E, Asghari G et al. (2015) Consumption of sugar sweetened beverage is associated with incidence of metabolic syndrome in Tehranian children and adolescents. Nutr Metab (Lond) 12, 25.

40. Gibney MJ, Forde CG, Mullally D et al. (2017) Ultra-processed foods in human health: a critical appraisal. Am J Clin Nutr 106, 717-724.

41. Monteiro CA, Cannon G, Moubarac JC et al. (2018) Ultra-processing. An odd 'appraisal'. Public Health Nutr 21, 497-501.

42. Rothman KJ, Greenland S \& Lash TL (2011) Modern Epidemiology, 3rd ed. Porto Alegre, RS: Artmed.

43. Szklo M \& Nieto FJ (2007) Epidemiology: Beyond the Basics, 2nd ed. Sudbury, MA: Jones and Bartlett Publishers.

44. Willett WC (1994) Future directions in the development of food-frequency questionnaires. Am J Clin Nutr 59, 1 Suppl., 171S-174S.

45. Ministry of Health of Brazil (2014) Dietary Guidelines for the Brazilian Population. Brasília, DF: Ministério da Saúde. 